\title{
Diseño y construcción de una máquina Dip Coater para la obtención de recubrimientos de $\mathrm{SiO} 2$ mediante la técnica sol-gel
}

\author{
Julieth Alejandra Ospina-Calderón ${ }^{1}$ \\ Universidad Antonio Nariño \\ julieospina@uan.edu.co \\ Camila Alejandra Lota-Mendoza ${ }^{2}$ \\ Universidad Antonio Nariño \\ clota99@uan.edu.co
}

Brayan Stick Betin-Bohórquez ${ }^{3}$

Universidad Antonio Nariño

brayanstickbetin@gmail.com

Carlos Arturo García-Gómez ${ }^{4}$

Universidad Antonio Nariño

carlosarturo81@uan.edu.co

Rolando Javier Rincón-Ortiz ${ }^{5}$

Universidad Antonio Nariño

rolrincon@uan.edu.co

Daniel Llamosa-Pérez ${ }^{6}$

Universidad Antonio Nariño

dllamosa@uan.edu.co

DOI: https://doi.org/10.21158/23823399.v7.n0.2019.2577

Fecha de recepción: 12 de septiembre de 2019

Fecha de aprobación: 05 de diciembre de 2019

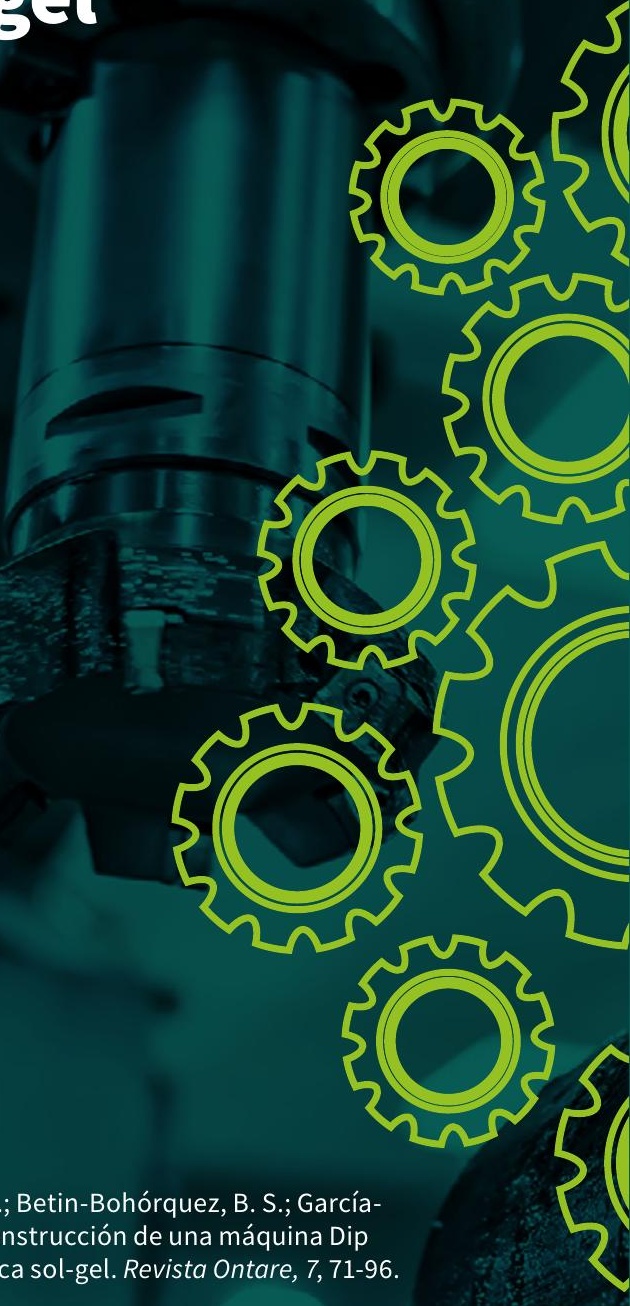

Cómo citar este artículo: Ospina-Calderón, J. A.; Lota-Mendoza, C. A.; Betin-Bohórquez, B. S.; García-

Gómez, C. A.; Rincón-Ortiz, R. J.; Llamosa-Pérez, D. (2019). Diseño y construcción de una máquina Dip Coater para la obtención de recubrimientos de SiO2 mediante la técnica sol-gel. Revista Ontare, 7, 71-96.

DOI: https://doi.org/10.21158/23823399.v7.n0.2019.2577

\footnotetext{
${ }^{1}$ Ingeniería industrial - Universidad Antonio Nariño. Ingeniería en control y automatización industrial - Universidad Antonio Nariño. ORCID: https://orcid.org/0000-0002-3475-3305

${ }^{2}$ Estudiante de Bioquímica - Universidad Antonio Nariño. ORCID: https://orcid.org/0000-0001-6732-0998

${ }^{3}$ Estudiante de Bioquímica - Universidad Antonio Nariño. ORCID: https://orcid.org/0000-0001-7875-808X

${ }^{4}$ Ingeniero Electrónico - Universidad Pedagógica y Tecnológica de Colombia. Magíster en Ingeniería Electrónica Procesamiento Digital de Señales (DSP) - Pontificia Universidad Javeriana. ORCID: https://orcid.org/0000-0002-0874-3795

${ }^{5}$ Químico - Universidad Nacional de Colombia. Doctor en Ciencias Química - Universidad Nacional de Colombia. Doctor en Química Molecular y Ciencia de Materiales - Universitat Jaume I. ORCID: https://orcid.org/0000-0001-5968-533X

${ }^{6}$ Ingeniero Físico - Universidad Nacional de Colombia. Magíster en Materiales Avanzados y Nanotecnologías - Universidad Autónoma De Madrid.

Doctor en Materiales Avanzados y Nanotecnologías - Universidad Autónoma De Madrid. ORCID: https://orcid.org/0000-0002-8310-6104
} 


\section{RESUMEN}

El presente documento presenta el diseño, la construcción y el análisis de resultados de una máquina denominada Dip Coater, un equipo empleado para la obtención de recubrimientos sobre diversos materiales, cuya técnica se basa en la inmersión y la extracción controlada de un sustrato en una solución. El trabajo da cuenta de la construcción de este equipo, que emplea, principalmente, un motor PAP y un microcontrolador Arduino Mega 2560, en el laboratorio de nanomateriales de la Universidad Antonio Nariño. El desarrollo del equipo tuvo cuatro fases: diseño, proptotipado, construcción y validación. El Dip Coater ofrece un control ajustable de parámetros tales como la velocidad de inmersión/extracción, los tiempos de inmersión y secado, el número de ciclos de inmersión y el desplazamiento de inmersión, por lo que en esta investigación se evaluó la respuesta interacción usuariomáquina y el comportamiento de la velocidad de extracción con diferentes masas. La reproducibilidad de los recubrimientos se estudió con el cambio de masa de estos en función de la velocidad de extracción. También se muestra la caracterización morfológica y físico-química de un recubrimiento solgel de $\mathrm{SiO} 2$ con propiedades anticorrosivas que pueden ser ajustadas por los parámetros del equipo Dip Coater. Finalmente, dentro de este proyecto logró diseñar y construir un instrumento científico competitivo que reúne características similares a las de los equipos comerciales.

Palabras clave: Dip Coater; dip coating; microcontrolador; motor PAP; interface HMI; caracterización morfológica; caracterización físico-química. 


\section{Design and construction of a Dip Coater for obtaining SiO2 coatings using the sol-gel technique}

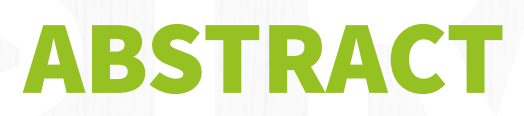

This document presents the design, construction, and analysis of results of the Dip Coater machine, a piece of equipment that is used to obtain thin film on various materials, using a technique that is based on the controlled immersion and extraction of a substrate into a solution. The work reports on the construction of this piece of equipment, which mainly uses a PAP engine and an Arduino Mega 2560 microcontroller, carried out in the nano-materials laboratory of the Antonio Nariño University, and that involved four phases: design, prototyping, construction, and validation. The Dip Coater offers an adjustable control of parameters: immersion/extraction speed, immersion and drying times, number of immersion cycles, and immersion displacement; so in this research, we evaluated the user-machine interaction response and the behavior of the extraction speed with different masses. The reproducibility of the coatings was studied as the mass changes according to the speed of extraction. The morphological and physical-chemical characterization of a sol-gel $\mathrm{SiO} 2$ coating is also shown, together with the anti-corrosive properties that can be adjusted by the parameters of the Dip Coater. Finally and within this project, it was possible to design and build a competitive scientific instrument with similar characteristics to those of the commercial equipment.

Keywords: Dip Coater; dip coating; microcontroller; PAP engine; HMI interface; morphological characterization; physical-chemical characterization. 


\section{Desenho e construção de uma máquina Dip Coater para a obtenção de recoberturas de SiO2 mediante o técnica sol-gel}

\section{RESUMO}

O presente documento apresenta o desenho, a construção e a análise de resultados de uma máquina denominada Dip Coater, empregada para a obtenção de recoberturas sobre diversos materiais, cuja técnica se baseia na imersão e a extração controlada de um substrato em uma solução. 0 trabalho apresenta a construção desta máquina, que emprega, principalmente, um motor PAP e um microcontrolador Arduino Mega 2560, no laboratório de nanomateriais da Universidade Antonio Nariño. O desenvolvimento da máquina teve quatro fases: desenho, prototipado, construção e validação. A Dip Coater oferece um controle ajustável de parâmetros tais como a velocidade de imersão/extração, os tempos de imersão e secado, o número de ciclos de imersão e a deslocação de imersão. Nesta pesquisa se avaliou a resposta interação usuário-máquina e o comportamento da velocidade de extração com diferentes massas. A reprodutividade das recoberturas estudou-se com a mudança de massa destas em função da velocidade de extração. Também se mostra a caracterização morfológica e físico-química de uma recobertura sol-gel de $\mathrm{SiO} 2 \mathrm{com}$ propriedades anticorrosivas que podem ser ajustadas pelos parâmetros da máquina Dip Coater. Finalmente, dentro deste projeto se conseguiu desenhar e construir um instrumento científico competitivo que reúne características similares às das máquinas comerciais.

Palavras-chave: Dip Coater; dip coating; microcontrolador; motor PAP; interface HMI; caracterização morfológica; caracterização físico-química. 


\section{Conception et construction d'un appareil Dip Coater via la technique sol-gel pour l'obtention de revêtements SiO2}

\section{RÉSUMÉ}

Ce document présente la conception, construction et analyse des résultats d'un appareil nommé Dip Coater utilisé pour l'obtention de revêtements sur divers matériaux dont la technique se base sur l'immersion et l'extraction contrôlée d'un substrat en solution. Les travaux rendent compte de la construction, dans le laboratoire de nanomatériaux de l'Université Antonio Nariño, de cet appareil munit d'un moteur PAP et d'un microcontrôleur Arduino Mega 2560. La processus de création de cet équipement s'est déroulé en quatre phases: conception, prototypage, construction et validation. Le Dip Coater offre un contrôle de paramétrage tels que la vitesse d'immersion / extraction, les temps d'immersion et de séchage, le nombre de cycles d'immersion et le déplacement par immersion. Nous avons de plus, lors de cette investigation, évalué la réponse d'interaction de l'utilisateur avec la machine et le comportement de la vitesse d'extraction de différentes masses. La reproductibilité des revêtements et leur changement de masse ont ici été analysés en fonction de la vitesse d'extraction. Une caractérisation morphologique et physicochimique d'un revêtement sol-gel $\mathrm{SiO} 2$ aux propriétés anticorrosives pouvant être ajustées via les paramètres de l'équipement Dip Coater sera également présentée. Enfin, ce projet a permis de concevoir un instrument scientifique compétitif aux caractéristiques similaires à celles des appareils disponibles sur le marché.

Mots clés: Dip Coater; revêtement par immersion; microcontrôleur; Moteur PAP; Interface IHM; caractérisation morphologique; caractérisation physico-chimique.. 


\section{Introducción}

La técnica dip coating se emplea tanto en la industria como en la academia con fines de investigación. Esta técnica se basa en la inmersión y la extracción de un sustrato en una solución de forma controlada y precisa, con el propósito de depositar en él una capa de material (Biolin Scientific, s. f.a). En la figura 1 se muestra el proceso de depósito de un recubrimiento, el cual presenta las siguientes etapas: a) sumergir el sustrato - velocidad y longitud de inmersión-; b) esperar hasta que la superficie de la solución llegue a un estado de reposo - tiempo de inmersión-; c) levante la de la muestra con una velocidad determinada - velocidad de extracción-; y d) se forma un recubrimiento uniformemente delgado después de eliminar el exceso de solución del objeto mediante un tratamiento térmico.

Los factores que contribuyen a la calidad, la uniformidad y la reproducibilidad en la fabricación de los recubrimientos son extensos. Así, principalmente, se debe tener en cuenta la superficie inicial del sustrato, el tiempo de inmersión, la velocidad de extracción, el número de ciclos del proceso, la composición, la concentración y la temperatura de la solución, así como las condiciones ambientales del entorno (Biolin Scientific, s. f.b).

Esta técnica presenta amplias ventajas. Entre estas se encuentran, por ejemplo, el recubrimiento de doble cara: ambos lados se pueden recubrir en un solo proceso; una menor pérdida de líquido de recubrimiento: la cantidad de líquido de recubrimiento utilizada es menor que la de los recubrimientos por rotación -spin coating - o por pulverización - sputtering-; es compatible con todas las formas de sustratos: incluso los objetos de trabajo con textura, incluidos los tableros de ensamblaje, pueden recubrirse; el enfoque es rentable: a diferencia de los métodos de procesamiento en seco, no se requieren instalaciones costosas, como, por ejemplo, hornos de vacío; por último, la simplicidad del equipo necesario, etc. (Chaki, Mahato, Malek y Deshpande, 2017).

Debido a que la técnica de dip coating es simple y de fácil control de sus parámetros, le otorga ventajas en los laboratorios dedicados al recubrimiento y el procesamiento de materiales para diferentes aplicaciones. Sin embargo, 
actualmente en Colombia no existe una industria en la producción de equipos para la fabricación de recubrimientos por inmersión dip coating. La alternativa que existe hoy en el nivel comercial es la importación, por medio de fabricantes tales como Nadetech Innovations de España (Nadetech Innovations, s. f.), MTI Corporation de California, Estados Unidos, o Holmarc Opto-Mechatronic PVT. LTD. de la India, entre otras, lo que incrementa el valor comercial del equipo (Biolin Scientific, s. f.c).

Figura 1. Esquema ilustrativo de las etapas de la técnica dip coating

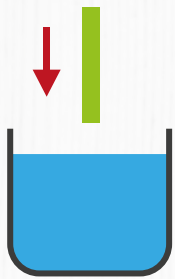

a.

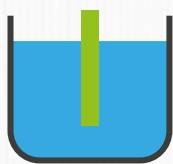

b.

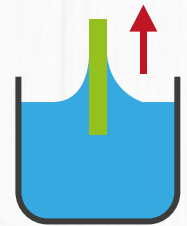

C.

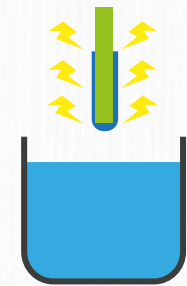

d.

Fuente. Elaboración propia.

Existen diversos reportes en la fabricación de equipos de inmersión para laboratorio. Estos equipos presentan elementos comunes tales como guías lineales, sistema de poleas, varillas aceradas, motores CC/PAP y sistemas eléctricos/electrónicos controlados, principalmente, por Arduino ${ }^{\oplus}$. Sin embargo, cada uno se diferencia en su diseño por la aplicación que se le dará o los procesos de síntesis, como, por ejemplo, el tamaño de pieza o la velocidad de inmersión (Baptista-Machuno et al., 2015; Ventura, Muñoz, Hernandez, Cristoph y Cortez, 2017).

Los recubrimientos que se realizaron en este trabajo fueron sintetizados por el método de inmersión en soles inorgánicos, también llamada síntesis solgel. Esta técnica se usa ampliamente en la ciencia de los materiales con el fin de crear recubrimientos protectores, recubrimientos ópticos, recubrimientos cerámicos y superficies similares (Hernández-Sánchez, 2013). Este método de síntesis se inicia con la hidrólisis de un precursor líquido - sol-, el cual se somete a una policondensación a fin de obtener gradualmente un gel. El proceso sol-gel se empleó en esta investigación para el desarrollo de capas de protección en la corrosión en metales. 


\section{Metodología}

El diseño conceptual para la construcción de la máquina Dip Coater y su desempeño se muestra en la figura 2 y se describe a continuación.

Figura 2. Prototipo inicial Dip Coater
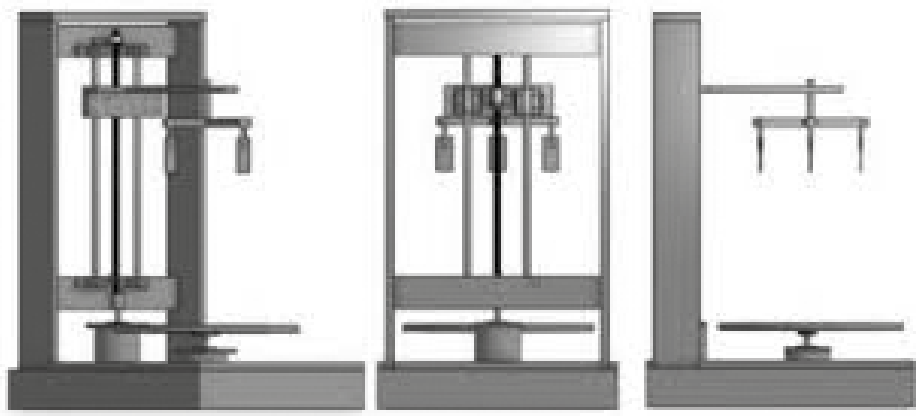

Fuente. Elaboración propia.

\subsection{Fase de diseño}

Esta fase consiste en la búsqueda de referencias bibliográficas sobre diseños de máquinas Dip Coater a nivel académico y comercial, con el propósito de conocer y analizar las variables técnicas y las restricciones del diseño electromecánico que interfieren en el desempeño de la máquina; y con la finalidad de realizar una simulación del diseño mecánico en CAD, del sistema de inmersión, con un desplazamiento vertical de la máquina de $290 \mathrm{~mm}$ y una velocidad de inmersión-extracción de 0,6 cm/min hasta $60 \mathrm{~cm} / \mathrm{min}$. 


\subsection{Fase de prototipado}

Se desarrolla la búsqueda y la compra de los materiales necesarios para la creación del prototipo de la estructura mecánica de la máquina Dip Coater, con el objeto de realizar pruebas de funcionamiento en condiciones variables, como, por ejemplo, el peso de las muestras -menores $1 \mathrm{~kg}$ en total-, o la velocidad de desplazamiento ascendente-descendente. También se desarrolla el software de control de los parámetros de funcionamiento de la máquina Dip Coater en Nextion Editor. La finalidad de esta fase es analizar el funcionamiento del prototipo que se muestra en la figura 2, con el fin de hallar mejoras estructurales o de control para implementar en la versión definitiva de la máquina Dip Coater.

\subsection{Fase de construcción de la máquina}

Una vez realizado y verificado el funcionamiento del prototipo, se procede a la fabricación de la versión final de la máquina Dip Coater, la cual se muestra en la figura 3, con la implementación estructural y electromecánica. Además, se integra el software de control con el objetivo de realizar pruebas de funcionamiento eléctrico y mecánico en el producto terminado.
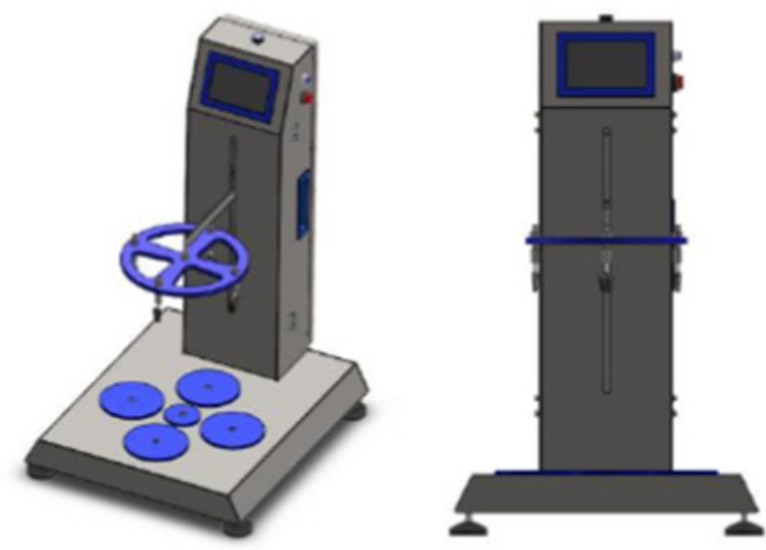

Fuente. Elaboración propia. 


\subsection{Fase de validación}

Finalmente, se realizó el registro lógico de software del programa de control de la máquina Dip Coater. En la figura 4 se muestra una fotografía del estado final de la máquina. Se realizaron diferentes pruebas de validación, evaluando la respuesta de la interacción usuario-máquina y el comportamiento del sistema sin carga y con carga de $0,75 \mathrm{~kg}$ y $1 \mathrm{~kg}$, teniendo en cuenta, sobre todo, la velocidad de extracción en diferentes valores. Además, se realizaron pruebas de masa a los recubrimientos con el fin de analizar la reproducibilidad de esta y el cumplimiento de la proporcionalidad que existe entre el aumento de la velocidad de extracción y el aumento de la masa del recubrimiento.

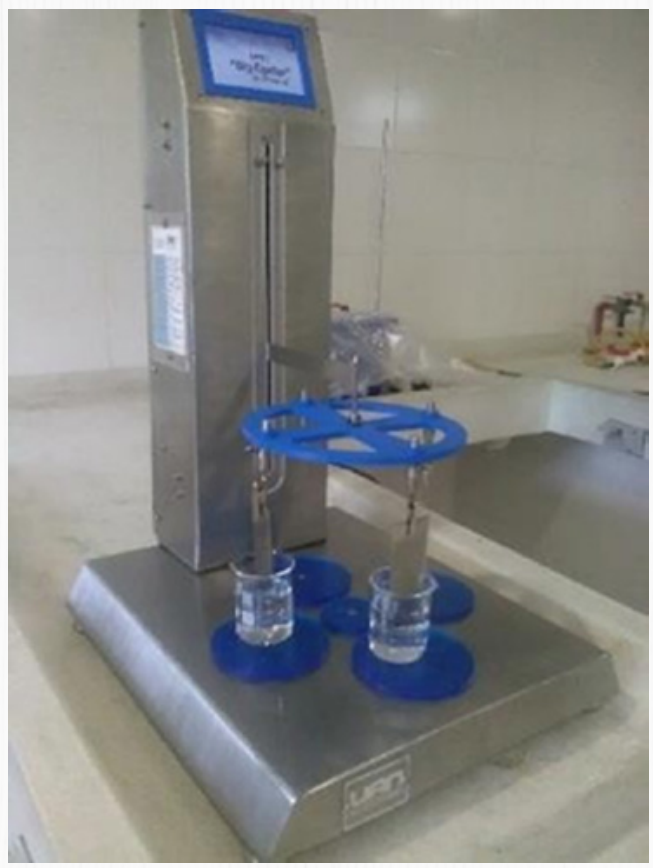

Fuente. Elaboración propia. 


\section{Construcción y validación de la máquina DIP Coater}

\subsection{Materiales y componentes de construcción}

A continuación, se enlistan los materiales empleados para la máquina DIP Coater.

- Motor PAP bipolar. Genera el movimiento vertical del proceso de inmersión.

- Fuente conmutada. Fuente switcheada encargada de la alimentación del sistema.

- Varilla trapezoidal. Varilla roscada, en acero inoxidable, de cuatro hilos y un acople en bronce para el movimiento vertical del sistema de inmersión.

- Varillas en acero plata y soportes lineales. Dos varillas lisas encargadas de soportar y guiar el peso del movimiento vertical del sistema. Estas varillas están sujetadas al sistema por medio de los cuatro soportes lineales.

- Rodamientos. Dos rodamientos lineales encamisados Sc8uu para la transmisión del movimiento en el sistema vertical y un rodamiento guía con soporte Kp08 para el husillo.

- Camisa tuerca trapezoidal. Elemento impreso en PLA para la unión de la tuerca del tornillo trapezoidal con el sistema de portasustratos.

- Carcasa. Estructura del sistema de inmersión en acero inoxidable AISI304, tipo B, calibre 14.

- Driver DRV8825. Driver de corriente para el control del motor PAP. 
- Panel táctil. Pantalla LCD de 5" a 5 V. Proporciona la interfaz HMI del sistema mediante el uso del puerto serial para la adquisición y visualización de datos.

- Controlador. Tarjeta de desarrollo Arduino Mega, construida con un microcontrolador Atmega2560 que posee pines de entradas y salidas $-\mathrm{E} / \mathrm{S})$ - , analógicas y digitales. Esta tarjeta es programada en un entorno de desarrollo que implementa el lenguaje Processing/Wiring.

En la figura 5 se muestra en detalle el diseño final aprobado de la máquina Dip Coater, de manera que se relaciona cada una de sus partes.

\section{Figura 5. Partes de la máquina Dip Coater}

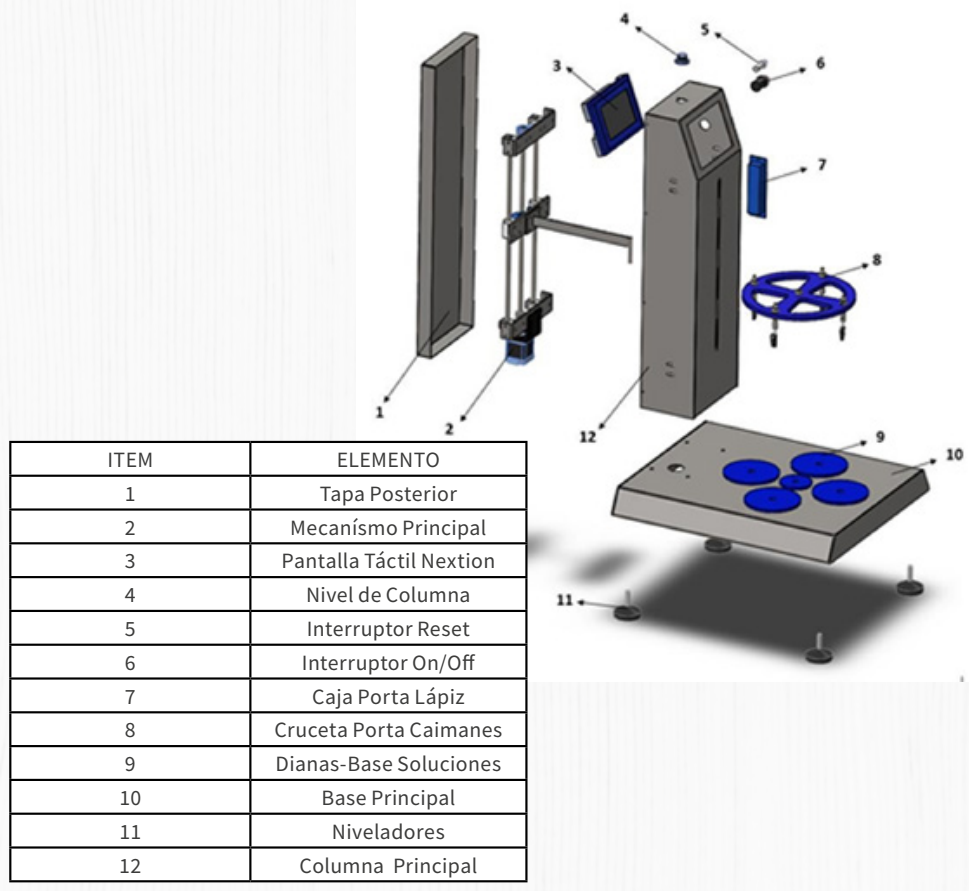

Fuente. Elaboración propia. 


\subsection{Código para el software del sistema de inmersión Dip Coater}

El código del programa está compuesto por el diseño de la interfaz HMI y la programación del microcontrolador. La interfaz usa un display Nextion touch TFT de 5", lo cual proporciona un ambiente gráfico amigable, práctico y sencillo. Este dispositivo se comunica con el microcontrolador a través del puerto serial, con una velocidad de transmisión de 9500 baudios. Además, posee un software de programación gratuito llamado Nextion Editor.

Ahora bien, se elaboró una interfaz que comprende una serie de botones tanto para que el usuario manipule el sistema y envíe instrucciones al microcontrolador como para que navegue entre páginas. En la figura 6 se muestran algunas de estas páginas.

Figura 6. Interfaz gráfica del equipo Dip Coater
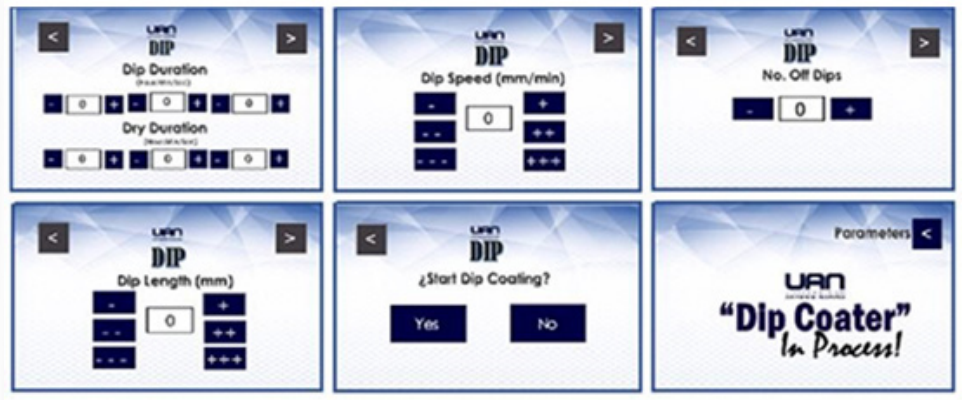

Fuente. Elaboración propia.

\subsection{Desempeño experimental}

Se realizaron diferentes pruebas a la máquina con el fin de conocer la respuesta del sistema de control de velocidad del motor PAP. La velocidad de inmersión/extracción real de la máquina se determinó por análisis de videos mediante el software Tracker. Este software permitió realizar el seguimiento manual y automático de objetos con superposiciones, generando datos de posición, velocidad y aceleración en función del tiempo. Del mismo modo, se realizaron pruebas aleatorias del sistema con cargas de $1 \mathrm{~kg}$ y $0,75 \mathrm{~kg}$ en diferentes velocidades de extracción. Para cada prueba se realizaron tres 
videos, con lo que se obtuvo el promedio y la desviación estándar de la posición, la velocidad y la aceleración. Los valores medios fueron comparados con los valores introducidos por el usuario en la máquina.

\subsection{Producción y caracterización de películas anticorrosivas de $\mathrm{SiO} 2$}

A fin de demostrar que la máquina cumple con su función principal de producir recubrimientos uniformes y de interés, se sintetizó una dispersión coloidal - sol-empleando una ruta de síntesis híbrida catalizada por ácido, y como precursores para el $\mathrm{SiO} 2$ el tetraetil ortosilicato -TEOS, $99 \%$, (OC2H5)4-y el metiltrietoxisiloano - MTES, $98 \%$, CH3Si (OC2H5)3-, en una proporción molar de TEOS/MTES $=40 / 60$. El medio de reacción empleado fue etanol absoluto -EtOH, $100 \%$-. Se empleó ácido acético - AcAcet-y la hidrólisis fue conducida por una disolución de ácido nítrico $0,1 \mathrm{~N}$, en la que se incorporó el agua requerida para el proceso de hidrólisis. La mezcla de reacción se mantuvo en reflujo durante una hora a $40{ }^{\circ} \mathrm{C}$, homogeneizada mediante agitación magnética.

Una vez sintetizado el sol se prepararon los sustratos o piezas a recubrir. Se utilizaron sustratos rectangulares de vidrio y hierro. En el caso de los sustratos en acero fueron pulidas por un método abrasivo con papel de lija -tamaño de grano de 200 hasta 1500-a fin de eliminar posibles capas corrosivas. Todos los sustratos fueron sometidos por 15 minutos a un baño ultrasónico.

En el propósito de contener las soluciones en el proceso de inmersión/ extracción se usaron recipientes en vidrio y en acero inoxidable, previamente limpiados con alcohol industrial en ultrasonido por $15 \mathrm{~min}$.

La velocidad de inmersión/extracción se varió de 0,6-60 cm/min. En cambio, los parámetros, como, por ejemplo, el número de ciclos de inmersión -un ciclo-, el tiempo de inmersión -1 min-, la longitud de inmersión y el tratamiento térmico $-1200 \mathrm{C}$ a $2 \mathrm{~h}-$, se mantuvieron constantes durante todo el experimento. En la figura 7 se muestra el equipo con un sustrato a punto de ser recubierto. En el vaso de precipitados se encuentra el sol de $\mathrm{SiO} 2$ previamente sintetizado. 
Se empleó una balanza analítica de marca Adam ABL 225 y legibilidad de $0,01 \mathrm{mg}$ para medir las masas de los recubrimientos en el sustrato en función de la velocidad de extracción. La morfología de los recubrimientos se observó por microscopía electrónica de barrido -SEM-. La composición química se identificó por medio de espectroscopia de dispersión de energía de rayos $\mathrm{X}$ EDS-. Estas medidas se realizaron en un microscopio de barrido de electrones JEOL, modelo JSM 6490-LV. Para determinar el espesor de los recubrimientos en función de la velocidad de extracción se realizaron estudios de perfilometría en el borde del recubrimiento empleando el equipo perfilómetro mecánico Veeco, modelo Dektak 150. Finalmente, las propiedades protectoras de los recubrimientos se evaluaron mediante espectroscopia de impedancia electroquímica -EIS-, realizada en un potenciostato Autolab PGSTAT 302 controlado por el software NOVA 1,6.

Figura 7. Proceso de inmersión

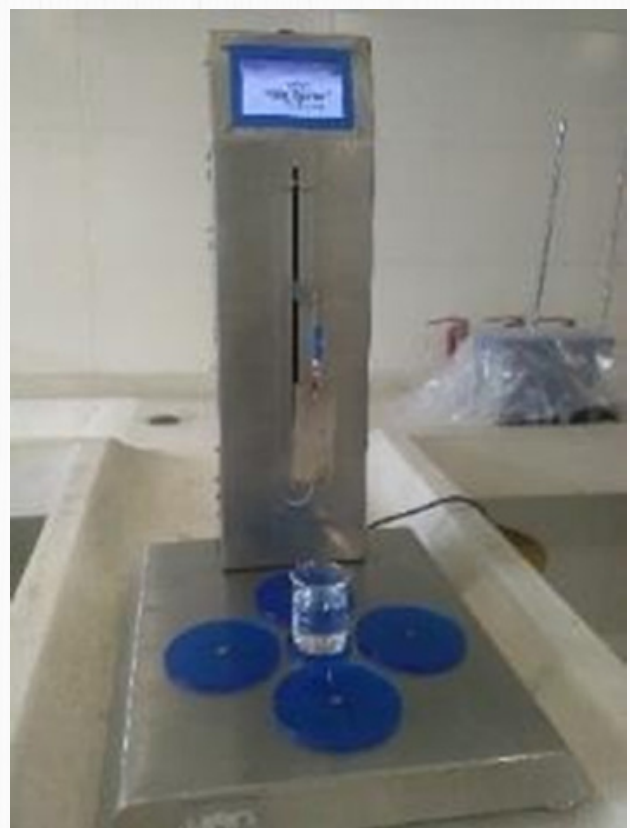

Fuente. Elaboración propia. 


\section{Resultados y discusión}

\subsection{Comportamiento de velocidad de extracción}

Empleando el software Tracker se obtuvieron los datos de posición de la muestra en función del tiempo. La velocidad de extracción varió de 0,6 a 6 $\mathrm{cm} / \mathrm{mim}$. En la tabla 1 se muestran a modo de ejemplo los resultados de las tres réplicas para la velocidad de extracción de $0,6 \mathrm{~cm} / \mathrm{min}$, y en la figura 8 se muestran las gráficas de estos datos. Además, se muestra la ecuación de la línea de tendencia para cada experimento. En la tabla 2 se presentan los resultados del análisis estadístico de los datos anteriores.

\section{Tabla 1. Datos obtenidos de tres videos analizados por Tracker 70}

\begin{tabular}{|c|c|c|c|c|c|}
\hline \multicolumn{2}{|c|}{ Ensayo 1 } & \multicolumn{2}{c|}{ Ensayo 2 } & \multicolumn{2}{c|}{ Ensayo 3 } \\
\hline $\mathbf{t}-$ & $\begin{array}{c}\mathbf{y}- \\
\mathbf{m m}-\end{array}$ & $\begin{array}{c}\mathbf{t}- \\
\mathbf{s}-\end{array}$ & $\begin{array}{c}\mathbf{y}- \\
\mathbf{m m}-\end{array}$ & $\begin{array}{c}\mathbf{t}- \\
\mathbf{s}-\end{array}$ & $\begin{array}{c}\mathbf{y}- \\
\mathbf{m m}-\end{array}$ \\
\hline 0,00 & 0,09 & 0,00 & 0,19 & 0,00 & 0,19 \\
\hline 71,2 & 9,68 & 69,2 & 9,46 & 70,0 & 9,89 \\
\hline 171, & 19,5 & 172 & 18,9 & 174 & 19,6 \\
\hline 260, & 29,1 & 262 & 28,7 & 262 & 29,1 \\
\hline 353, & 39,0 & 352 & 38,9 & 354 & 39,3 \\
\hline 435, & 49,1 & 439 & 48,7 & 445 & 49,1 \\
\hline 540, & 58,0 & 538 & 58,5 & 534 & 58,8 \\
\hline
\end{tabular}

Fuente. Elaboración propia. 
Figura 8. Grafica de posición contra tiempo para una velocidad de extracción $6 \mathrm{~mm} / \mathrm{min}$

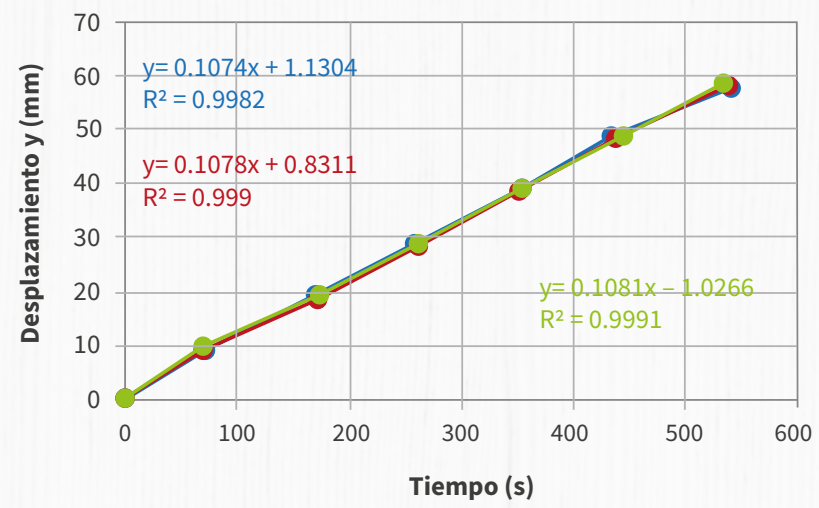

Fuente. Elaboración propia.

Tabla 2. Análisis estadísticos de los datos obtenidos de tres videos analizados por Tracker

\begin{tabular}{|c|c|}
\hline Ensayo $1-\tilde{v}-$ & $0,644 \mathrm{~cm} / \mathrm{min}$ \\
\hline Ensayo $2-\tilde{v}-$ & $0,649 \mathrm{~cm} / \mathrm{min}$ \\
\hline Ensayo $3-\tilde{v})-$ & $0,647 \mathrm{~cm} / \mathrm{min}$ \\
\hline Media & $0,647 \mathrm{~cm} / \mathrm{min}$ \\
\hline Error Relativo & $7,83 \%$ \\
\hline Varianza & 0,033 \\
\hline Desviación Estándar & 0,058 \\
\hline Ensayo $1-\tilde{v}-$ & $0,644 \mathrm{~cm} / \mathrm{min}$ \\
\hline Ensayo $2-\tilde{v}-$ & $0,649 \mathrm{~cm} / \mathrm{min}$ \\
\hline Ensayo $3-\tilde{v})-$ & $0,647 \mathrm{~cm} / \mathrm{min}$ \\
\hline Media & $0,647 \mathrm{~cm} / \mathrm{min}$ \\
\hline Error Relativo & $7,83 \%$ \\
\hline Varianza & 0,033 \\
\hline Desviación Estándar & 0,058 \\
\hline
\end{tabular}

Fuente. Elaboración propia. 
Este análisis se repitió para las velocidades de extracción $0,6,1,10,20,30$, 40,50 y $60 \mathrm{~cm} / \mathrm{min}$, repitiendo la prueba tres veces. En la tabla 3 se exponen los resultados.

Tabla 3. Comparación de las velocidades medias experimentales con las introducidas por el usuario.

\begin{tabular}{|c|c|c|c|}
\hline $\begin{array}{c}\text { Velocidad } \\
\text { deseada }-\mathbf{c m} / \\
\text { min- }\end{array}$ & $\begin{array}{c}\text { Velocidad media } \\
\text { obtenida } \\
-\mathbf{c m} / \text { min- }\end{array}$ & Error relativo & $\begin{array}{c}\text { Desviación } \\
\text { estándar }\end{array}$ \\
\hline 0,60 & 0,647 & $7,83 \%$ & 0,001 \\
\hline 1,00 & 1,06 & $6,36 \%$ & 0,007 \\
\hline 10,0 & 10,4 & $4,25 \%$ & 0,018 \\
\hline 20,0 & 20,1 & $0,45 \%$ & 0,303 \\
\hline 30,0 & 29,9 & $0,07 \%$ & 0,017 \\
\hline 40,0 & 40,1 & $0,11 \%$ & 0,152 \\
\hline 50,0 & 48,3 & $3,39 \%$ & 0,187 \\
\hline 60,0 & 56,8 & $5,38 \%$ & 0,107 \\
\hline
\end{tabular}

Fuente. Elaboración propia.

En la tabla 4 se muestran los resultados de las pruebas de inmersión realizadas con cargas de $0,75 \mathrm{~kg}$ y $1 \mathrm{~kg}$. Esto permitió comprobar el comportamiento del equipo y los posibles errores que se generan con el incremento de masa de la muestra.

Tabla 4. Comparación de los resultados estadísticos para diferentes velocidades cambiando la masa de la muestra.

\begin{tabular}{|c|c|c|c|c|}
\hline \multirow{2}{*}{ Carga - kg- } & $\begin{array}{c}\text { Velocidad } \\
\text { deseada }- \\
\mathbf{c m} / \mathbf{m i n}-\end{array}$ & $\begin{array}{c}\text { Velocidad } \\
\text { media } \\
\text { obtenida }- \\
\mathbf{m m} / \mathbf{m i n}-\end{array}$ & Error relativo & $\begin{array}{c}\text { Desviación } \\
\text { estándar }\end{array}$ \\
\hline \multirow{3}{*}{ Sin Carga } & 0,6 & 0,647 & $7,83 \%$ & 0,01 \\
\cline { 2 - 5 } & 30 & 29,9 & $0,07 \%$ & 0,017 \\
\cline { 2 - 5 } & 60 & 56,8 & $5,38 \%$ & 0,107 \\
\hline
\end{tabular}




\begin{tabular}{|c|c|c|c|c|}
\hline \multirow{3}{*}{0,75} & 0,6 & 0,651 & $8,47 \%$ & 0,06 \\
\cline { 2 - 5 } & 30 & 29,1 & $2,98 \%$ & 0,012 \\
\cline { 2 - 5 } & 60 & 53,6 & $10,66 \%$ & 0,363 \\
\hline \multirow{3}{*}{1} & 0,6 & 0,670 & $11,60 \%$ & 0,06 \\
\cline { 2 - 5 } & 30 & 30,3 & $0,99 \%$ & 0,309 \\
\cline { 2 - 5 } & 60 & 55,2 & $7,93 \%$ & 0,436 \\
\hline
\end{tabular}

Fuente. Elaboración propia.

Tabla 5. Masas de los recubrimientos en función de la velocidad de extracción

\begin{tabular}{|c|c|c|c|c|c|}
\hline \multirow{2}{*}{$\begin{array}{c}\text { Velocidad } \\
\text { Extracción } \\
\mathbf{- c m} \text { /min- }\end{array}$} & \multicolumn{5}{|c|}{ Masa del recubrimiento - mg- } \\
\cline { 2 - 6 } & Muestra 1 & Muestra 2 & Muestra 3 & $\begin{array}{c}\text { Promedio } \\
-\mathbf{m g}-\end{array}$ & $\begin{array}{c}\text { Desviación } \\
\text { estándar }\end{array}$ \\
\hline 0.1 & 2,31 & 2,40 & 2,10 & 2,27 & 0,15 \\
\hline 10 & 5,09 & 5,09 & 5,04 & 5,07 & 0,03 \\
\hline 20 & 7,76 & 7,88 & 8,00 & 7,88 & 0,12 \\
\hline 30 & 10,81 & 10,66 & 11,33 & 10,93 & 0,35 \\
\hline 40 & 13,41 & 12,77 & 13,05 & 13,08 & 0,32 \\
\hline 50 & 15,41 & 15,38 & 15,61 & 15,47 & 0,13 \\
\hline 60 & 17,86 & 17,89 & 17,62 & 17,79 & 0,15 \\
\hline
\end{tabular}

Fuente. Elaboración propia.

En la tabla 5 se presentan los resultados de las masas de los recubrimientos en función de la velocidad de extracción. Para este experimento se realizaron tres réplicas. Esta masa se calculó mediante la diferencia de masas de cada sustrato antes y después de cada inmersión. En la figura 9 se muestra la gráfica de la masa del recubrimiento en función de la velocidad de extracción; además, la ecuación de la línea de tendencia para estos valores. 
Figura 9. Masa del recubrimiento en función de la velocidad de extracción

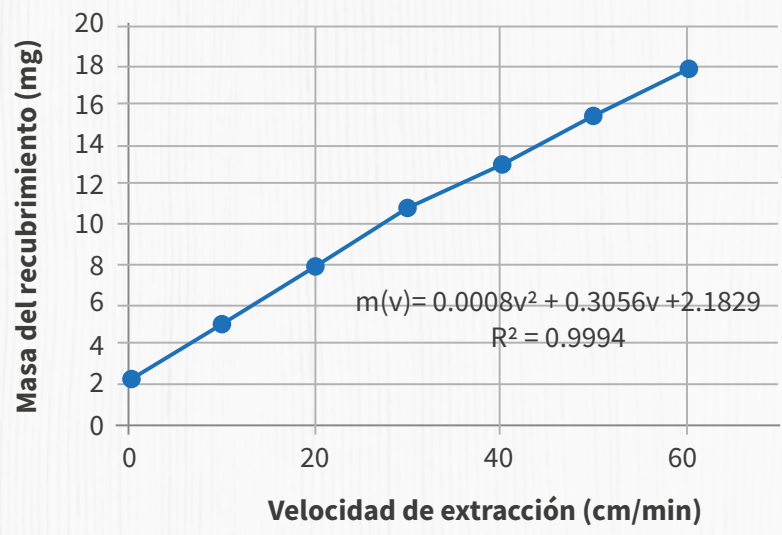

Fuente. Elaboración propia.

A fin de relacionar estos resultados con la morfología de los recubrimientos, en la figura 10 se exponen los perfiles de los recubrimientos a velocidades de extracción 20, 40 y $60 \mathrm{~cm} / \mathrm{min}$. En la parte superior derecha de esta figura se muestra la variación del espesor en función de la velocidad de extracción.

\section{Figura 10. Perfilometría de los recubrimientos a diferentes velocidades}

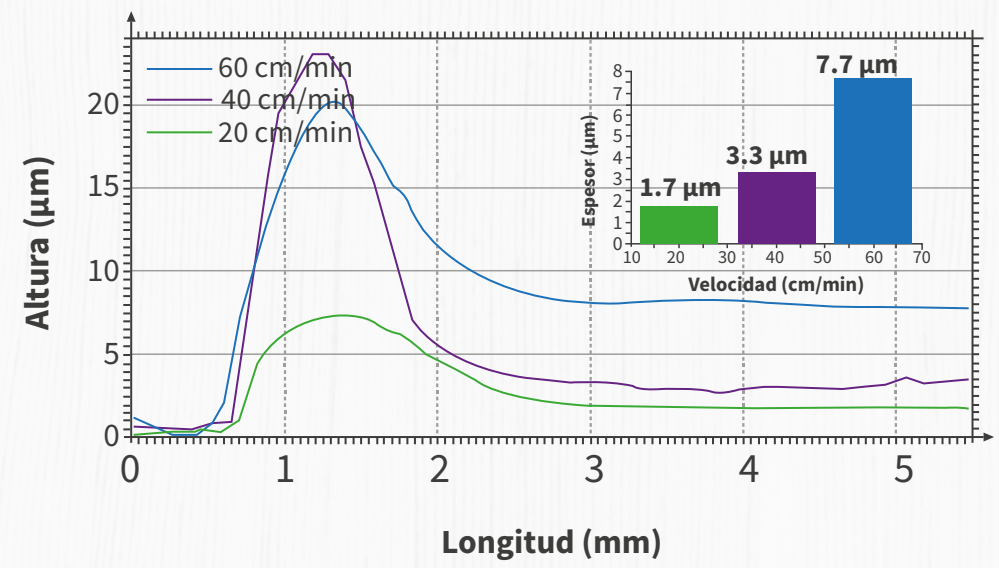

Nota: en el inciso superior se muestra la altura promedio

Fuente. Elaboración propia. 
Así mismo, en la figura 11 se observan las micrografías obtenidas por SEM para una velocidad de a) $20 \mathrm{~cm} / \mathrm{min}$ y b) $40 \mathrm{~cm} / \mathrm{min}$. En la parte inferior de cada micrografía se presentan los resultados de EDX de toda la micrografía mostrada, además de los porcentajes en peso obtenidos para los elementos de Fe y Si.

Figura 11. Imágenes de SEM y EDX para un recubrimiento con velocidad de extracción $20 \mathrm{~cm} /$ $\min$ y $40 \mathrm{~cm} / \mathrm{min}$
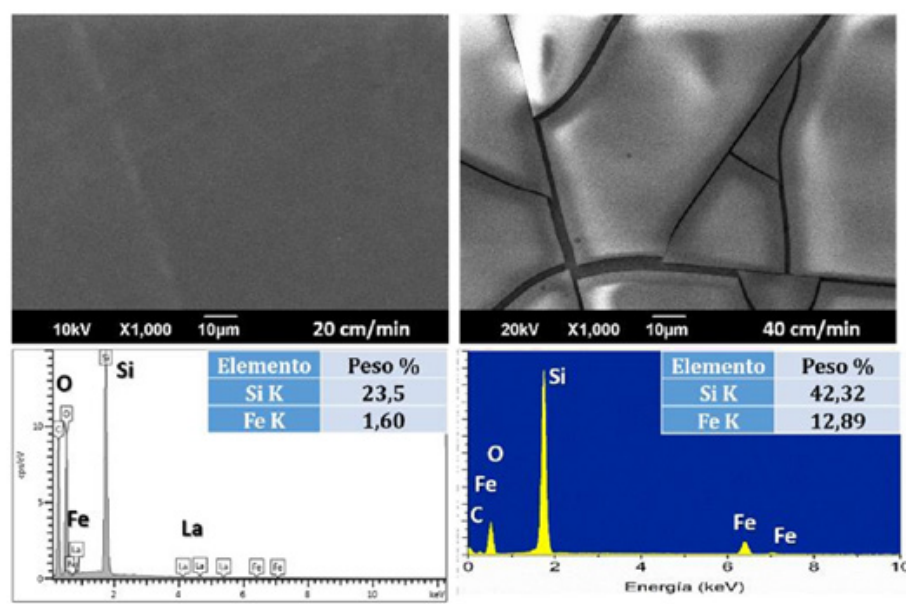

Fuente. Elaboración propia.

Finalmente, en la figura 12 se muestran los valores correspondientes a la resistencia de polarización (Rp) extraídos de los diagramas de Bode de EIS para bajas frecuencias. La Rp es la resistencia que el recubrimiento opone al paso de iones de la solución de trabajo al sustrato, impidiendo que esta sufra de corrosión. En términos generales, cuando un recubrimiento registra un alto valor de Rp se podrá concluir que es buen protector anticorrosivo. 
Figura 12. Valores de la resistencia a la polarización de los recubrimientos con diferentes velocidades de extracción

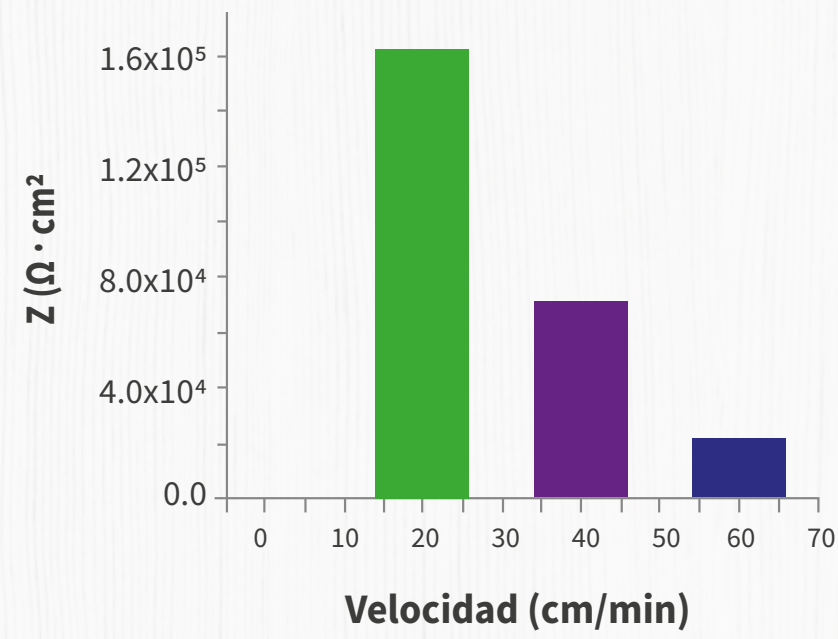

Fuente. Elaboración propia.

\section{Conclusiones}

Se diseñó y construyó un sistema programable de inmersión Dip Coater que trabaja de acuerdo con las siguientes variables de técnicas para la fabricación de recubrimientos: velocidades de inmersión y extracción desde $0,6 \mathrm{~cm} /$ min hasta $60 \mathrm{~cm} / \mathrm{min}$, un desplazamiento máximo de $29 \mathrm{~cm}$, un tiempo de inmersión y secado en un rango de 0 s a 24 h y diez ciclos máximos del proceso. El sistema permite sumergir y extraer diferentes tipos de sustratos tales como vidrio o metales, entre otros. Todas las variables ofrecidas se ajustan al objetivo general propuesto y son totalmente modificables por el usuario. Además, el sistema de desplazamiento y la velocidad se pueden ajustar con precisión por medio del uso del motor PAP controlado por el software y la interfaz HMI. Por otra parte, tiene la capacidad de realizar la inmersión con cuatro muestras con un peso total inferior de $1 \mathrm{~kg}$ y una dimensión por muestra mínima de 75 × 55 x 15 mm y máxima de $75 \times 55 \times 135 \mathrm{~mm}$. 
Se documentaron las variables técnicas asociadas al proceso de fabricación de recubrimientos homogéneos, las cuales fueron:

- superficie inicial del sustrato;

- composición y concentración de las soluciones;

- temperatura y el porcentaje de humedad del ambiente;

- velocidad de inmersión y extracción del sustrato;

- tiempo de inmersión y secado;

- número de procesos de inmersión;

- mínimas vibraciones.

Las tres primeras variables no se incluyeron en este trabajo, dado que están asociadas a los parámetros de composición de la solución, a la limpieza de los sustratos o a los parámetros ambientales. Por tanto, las variables incluidas en el diseño de la máquina Dip Coater corresponden a los cuatro últimos ítems mencionados en la lista.

Ahora bien, se diseñó y fabricó un dispositivo dedicado a la producción de películas delgadas por la técnica dip coating que se utilizarán en el laboratorio de nanomateriales de la Universidad Antonio Nariño. Los resultados de repetitividad de las pruebas de velocidad y de masa concluyen que el sistema tiene características que lo potencian como una herramienta de investigación para el estudio de materiales, la producción de recubrimientos de alta calidad y el fortalecimiento de la infraestructura científica de la universidad, ya que posibilita el crecimiento de las actividades de investigación y la docencia relacionadas con el tema de los nuevos materiales.

Se usó una pantalla táctil Nextion de 5" fabricada por Itead para la interface hombre-máquina; este dispositivo se programó mediante el software gratuito Nextion Editor, lo que proporciona flexibilidad en el momento de modificar y adaptar al hardware del sistema, además de la portabilidad de la máquina. También se incorporó la ventaja de fácil manipulación del equipo, con lo cual se hizo más independiente y se logró una mejor ubicación de este. 
Si bien en la actualidad existe una gran variedad de equipos comerciales de tipo dip coater, su costo y mantenimiento son elevados. Este proyecto logró diseñar y construir un instrumento científico competitivo que reúne características similares a las de los equipos comerciales. Las principales ventajas de este dispositivo son su costo, su facilidad de operación, su diseño robusto y estable, sin vibraciones y portátil, con piezas mecánicas de fácil adquisición y un software de código abierto. El desarrollo de esta máquina con el uso de la placa Arduino lo hace versátil, ya que se puede aplicar casi en cualquier instrumento que necesite ciertos procesos lógicos y permite hacer posibles mejoras futuras en el sistema.

\section{Agradecimientos}

Al Semillero de Nanomateriales de la Universidad Antonio

Nariño, por el apoyo financiero brindado por medio del proyecto interno de investigación 2017223. 


\section{Referencias}

Baptista-Machuno, L.; Oliveira, A.; Furlan, R.; Barbosa-Lima, A.; Morais, L. C.; Gelamo, R. (2015). Multilayer graphene films obtained by dip coating technique. Materials Research, 18(4), 775-780. DOI: https://doi. org/10.1590/1516-1439.005415

Biolin Scientific. (s. f. a). Dip Coater. Recuperado de https://bit.ly/30wozKg

Biolin Scientific. (s. f. c). Dip coaters: fabrication \& deposition of thin films. Recuperado de https://bit.ly/30B80Sr

Biolin Scientific. (s. f. b). Dip coating: measurements. Recuperado de https:// bit.ly/2CttiEH

Chaki, S. H.; Mahato, K. S.; Malek, T. J.; Deshpande, M. P. (2017). CuAlS 2 thin films-Dip coating deposition and characterization. Journal of Science: Advanced Materials and Devices, 2(2), 215-224. DOI: https://doi. org/10.1016/j.jsamd.2017.04.002

Hernández-Sánchez, M. Y. (2013). Síntesis de pigmentos cerámicos por métodos no convencionales: técnica sol-gel (Tesis de maestría). Universidad Nacional de Colombia, Colombia. Recuperado de https://bit.ly/2E9vwcK

Nadetech Innovations. (s. f.). Dipcoating. Recuperado de https://bit.ly/2ZOj2Q8

Ventura, S.; Muñoz, R.; Hernandez, A.; Cristoph, R.; Cortez, K. (2017). Instrumento de recubrimiento para deposición por inmersión (dip coating). Nanotecnia. Recuperado de https://bit.ly/39h1iju 